\title{
Geometrie dello sguardo nel paesaggio calabrese
}

\author{
Geometries of the gaze in the Calabrian landscape
}

\author{
Francesca Fatta ${ }^{\text {a }}$, Andrea Marraffa ${ }^{\text {b }}$, Claudio Patanè ${ }^{c}$ \\ Università degli Studi Mediterranea di Reggio Calabria, Reggio Calabria, Italy \\ affatta@unirc.it; ${ }^{\mathrm{b}}$ andrea.marraffa@unirc.it; ${ }^{\mathrm{c}}$ claudio.patane@unirc.it
}

\begin{abstract}
Having lost their function of sighting as an instrument of strategic control, inclusion and protection from presumed pirate invasions, the coastal towers of Calabria Ultra, represented in the Diary of Wonders of the end of the sixteenth century, called Codice Romano Carratelli, will act as the key and device of the gaze that links the land to the expanse of water. A vast geometric, precise and linear system that will connect, through the gaze, the "terracqueo landscape", unstable and multiform, continuously changing. The ninety-nine watercolour maps of the Codice are an immense heritage of clues, traces, geometries and measurements on which to think in order to bring to the surface of the earth, military tactics that have become latent in history as a palimpsest. The use of ancient and modern techniques of survey and graphic representation, want to accompany the contemporary traveler to turn his gaze towards new strategies of "reception", rather than aversion of a silent landscape, where merge and mix. The "stratigraphies of the gaze" are sections perpendicular to the "horizontal plane" of a "living" landscape from which routes, artefacts, signs, traces, fragments of history can be distilled for a widespread cultural regeneration of the territory. The experimental character of this research, recounted in these pages, lies in the application of an innovative strategy of communication and information, based on the creation of cultural routes structured in museums, widespread or located on the coastal landscape of Calabria.
\end{abstract}

Keywords: Calabria, Codice Romano Carratelli, coastal towers, cultural landscape, relief design.

It is precisely the act of drawing which forces the artist to watch the object in front of you, to dissect it with the eyes of the mind and put it back together. (John Berger)

\section{Introduzione alla ricerca: la rete delle torri costiere nel Codice Romano Carratelli}

Il Codice Romano Carratelli rappresenta un patrimonio di inestimabile valore documentale, non solo per la Calabria, ma anche per la documentazione cartografica del Mediterraneo. Riprendendo quanto asserito da Lucio Gambi, le antiche carte come il Codice sono da considerarsi "[...] oggetti che, quasi in termini dialettici col discorso scritto, riflettono con messaggi grafici i modi di pensare o interpretare la realtà materiale entro cui si svolge la nostra vita" (Gambi, 1984. p. 10). Secondo questa logica risulta affa- scinante studiarne non solo i contenuti, ma anche e soprattutto i vasti rapporti con la società che li ha prodotti: le ragioni che ne hanno determinato la nascita, il tipo di cultura che ha sostanziato la loro realizzazione, i diversi linguaggi con cui si sono espressi, l'impronta che hanno lasciato nella forma e nei gradi di comprensione delle realtà territoriali odierne.

Il disegno delle mappe storiche è divenuto inoltre un tema portante per i rinnovati studi sulla 
geografia fin dagli anni settanta del secolo scorso. In quegli anni il geografo francese Yves Lacoste scriveva, mettendo in perfetta luce il binomio geografia-potere, che la geografia serve innanzi tutto a fare la guerra e a organizzare i territori per meglio controllare gli uomini sui quali l'apparato statale esercita la sua autorità. La cartografia è stata, prima di ogni cosa, un sapere politico e militare; solo attraverso conoscenze di tipo geografico e cartografico si può pianificare il territorio e sfruttarlo in modo 'intraprendente'. La cartografia rappresenta dunque un insieme di conoscenze tutte finalizzate alla elaborazione di strategie e tattiche. E questo è il primo motivo per cui il Codice Romano Carratelli viene ideato, progettato e realizzato.

Oltre alla rappresentazione cartografica ufficiale delle città e del paesaggio, grandiosa e celebrativa, già alla fine del XVI secolo si andava diffondendo in Europa un disegno più specialistico finalizzato a descrizioni tecniche e destinato al funzionamento del territorio. Si tratta del disegno di un "mondo reale" che aveva la necessità di essere registrato in modo comprensibile. Così venne redatto il Codice Romano Carratelli: novantanove carte acquerellate in cui si distinguono i dati geografici caratterizzanti i tratti più significativi delle coste calabresi: corsi d'acqua, linee di costa, variazioni altimetriche e aggregati urbani compatti: cinte murarie, campanili, torri, architetture emergenti. Nel caso del Codice Romano Carratelli, le descrizioni puntuali e conseguenziali delle torri di difesa (presenti e future) riassumono un grande progetto di potenziamento difensivo delle coste. Il quadro che ne deriva può definirsi un fondamentale documento antropo-geografico della Calabria Ultra, il più antico e completo che si conosca. Questo tipo di carte può essere riferito al tracciato dei portolani o delle carte nautiche, i quali, sulla semplice definizione del contorno della costa e delle descrizioni essenziali del costruito, creano un voluto azzeramento degli apparati grafici di contorno, definendo una suggestione grafica moderna. Nel caso del Codice poi, si concretizza un aspetto inedito, ovvero un ordine di segni e una metodologia grafica che determinano una eleganza comunicativa unica. La coerenza descrittiva, il coordinamento nei colori (pochi ma robusti), l'impaginato elegante su carta che ha mantenuto nel tempo la sua meccanica (indeformabile e quindi inalterabile nella sua composizione) fa sì che l'intero documento possa definirsi Codice, proprio per la coerenza che esprime anche con un'unica grafia in corsivo maiuscolo/minuscolo. I rapporti dimensionali degli elementi rappresentati sono alquanto coerenti con il racconto delle tavole, si prescinde dalla scala grafica e si dà spazio alle forme e alle posizioni prevalenti dei manufatti. Il colore descrive il passaggio tra il rilievo dello stato di fatto e il progetto delle opere previste: in ocra rossa l'esistente, e in azzurro grigio ciò che è progetto. La scelta del colore è conseguenziale ad un pensiero moderno: la tinta di ciò che esiste riflette la sedimentazione dei segni antropici, il colore grigio blu è il segno della modernità che si inserisce nel contesto in modo coerente. Nel Codice, il disegno dei luoghi è realizzato con sistemi di rappresentazione misti, tra visioni prospettiche e schemi assonometrici, data la chiara impostazione militare, il punto di vista è posto in alto (a volo d'uccello) con veduta sempre dal mare verso la costa.

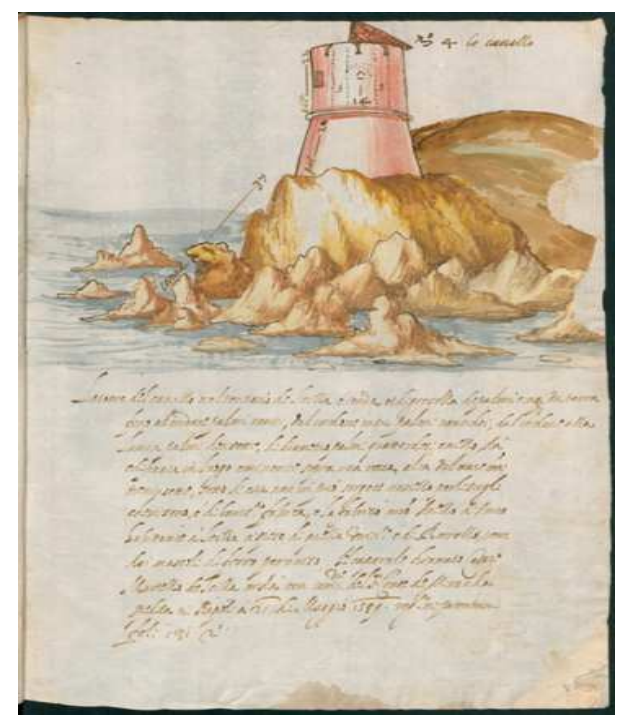

Fig. 1. Codice Romano Carratelli, Torre Lo Cavallo, disegnata e trascritta dall' anonimo autore, XVI secolo.

Sfogliando le tavole del Codice i luoghi forti descrivono un itinerario completo e puntuale delle coste calabresi che ci porta ad immaginare un 
"Grand Tour culturale". Questi luoghi, rappresentati con tecnica ad acquerello, segnano la presenza di manufatti architettonici che hanno avuto un ruolo fondamentale nella definizione del paesaggio urbano e rurale. Gli insediamenti costituiscono una rete di collegamenti sulle coste che rappresentano i capisaldi della difesa nel Mediterraneo legati da misure visive, da allineamenti e scorci calcolati. Ogni tavola rappresenta un luogo e fissa un intervallo calcolabile con la tavola successiva per il completamento di una geografia territoriale. I luoghi delle torri appaiono quindi come uno sfondo concreto e presente nel quale i manufatti si misurano. Spesso il taglio prospettico del disegno consente di raggiungere forme di integrazione tra architettura e natura, sempre accompagnate da note di carattere economico e costruttivo.

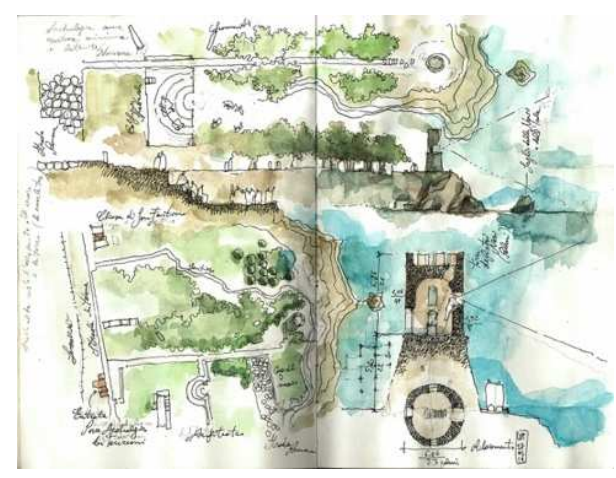

Fig. 2. Ruderi d'invenzione_stratigrafie dello sguardo. Rilevamento in modalità analogica disegno di C. Patanè. Inchiostro di china e acquarello su taccuino da viaggio, 2019.

Le qualità formali delle architetture, i materiali descritti e le tecniche di realizzazione tradiziona$\mathrm{li}$, insieme alle tipologie fissate dalle regole militari, ci restituiscono manufatti di grande semplicità e chiariscono ancora di più il profondo legame con la tradizione materiale costruttiva di questa terra.

Gli spunti di ricerca concreti che il Codice suggerisce, portano a immaginare itinerari tematici capaci di riprendere il racconto delle coste calabresi considerando quello che resta visibile (dei castelli, delle torri, delle antiche fortezze e degli insediamenti storici) immerso, mescolato e ab- bandonato in un contesto attuale che ingloba, somatizza, trasforma e abbandona. Ciascuno di noi, grazie ai fantastici acquerelli può trasformarsi in lettore/viaggiatore, portato a fare molte scoperte secondo itinerari possibili alla scoperta di "paesaggi culturali" affascinanti. In definitiva il Codice può dare un contributo concreto, non tanto alla difesa delle coste, quanto alla difesa di luoghi affascinanti ma insidiati da un presente che dimentica in fretta.

\section{Il progetto per un Grand Tour culturale}

Il progetto di ricerca si articola in una fase iniziale di ricognizione, rilievo e schedatura, utilizzando sia le tecniche classiche del rilievo diretto che le tecniche più avanzate del rilievo strumentale dei manufatti esistenti (Fig. 2). Le torri sono state documentate con il disegno dal vero, la fotocamera digitale, laser scanner e drone. La fase successiva riguarda la restituzione grafica tridimensionale mediante nuvola di punti e la modellazione parametrica delle torri presenti nel Codice (Fig. 3).

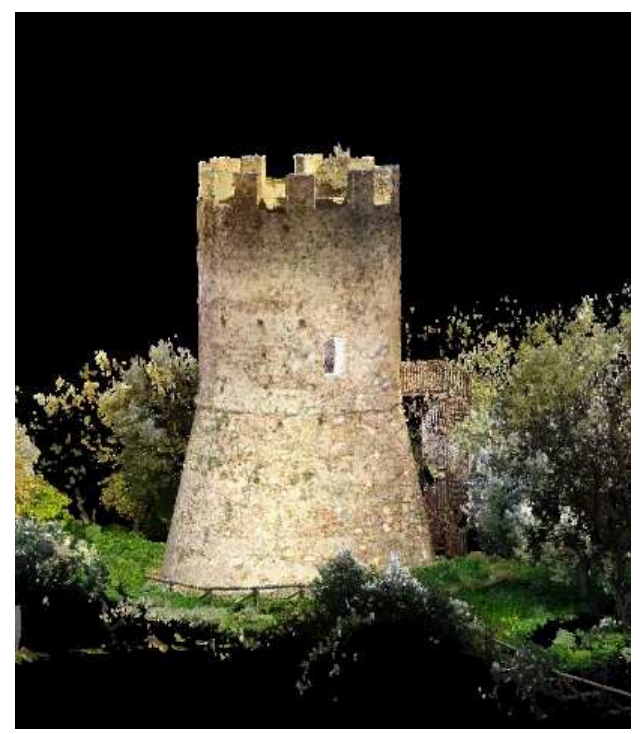

Fig. 3. Restituzione nuvola di punti della Torre Le Pietre Nere (Palmi-Reggio Calabria). Rilevamento effettuato con Laser Scanner FARO.

Sia la prima che la seconda fase sono state un momento di riflessione speculare sul concetto di "geometria dello sguardo" che investe l'intero 
progetto di difesa relativo al Codice e, in parallelo, sull'idea di "sguardo pittoresco" che riguarda il paesaggio contemporaneo. Esperienza questa, di una narrazione grafica dinamica, non più statica come in passato, in cui il disegno diviene atto progettuale e processo di forme in continuo divenire, piuttosto che di forme finite (Renato Bocchi, 2006). Ne sono scaturite da ciò delle "carte illustrate": Le stratigrafie dello sguardo e I ruderi d'invenzione (Fig. 4). Infine la fase di programmazione dei dati ottenuti determinerà il progetto, il cui carattere strategico, dalla scala locale a quella territoriale, ha come obiettivo quello di mettere in relazione un sistema puntuale di manufatti, esistenti e non, costituito dalle torri d'avvistamento, per renderle "chiavi" di un sistema di luoghi della cultura a carattere diffuso, che "avvista e accoglie" in forma tangibile, palpabile, quell'orizzonte fatto di mare o di terra, di storia, di memoria e di conoscenza. Quindi costruire, nel reale, spazi d'interazione virtuale per "un'immersione sensibile" dell'utente, che sarà coinvolto in maniera non più passiva ma interattiva, ludica e costruttiva. Partecipe con il corpo di un paesaggio vivo che "ci costruisce e ci ascolta" (Abalos, 2005).

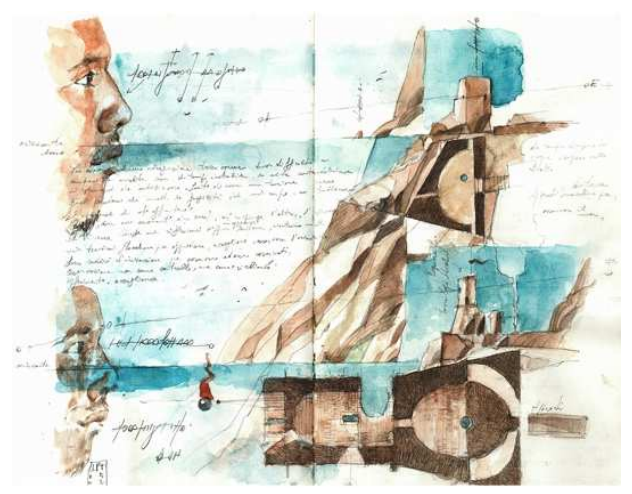

Fig. 4. Ruderi d'invenzione_stratigrafie dello sguardo, disegno di C. Patanè. Matita e acquarello su taccuino da viaggio, 2019.

\section{Dal rilievo digitale all'interpretazione se- mantica del paesaggio archeologico}

Il territorio descritto nelle tavole del Codice Romano Carratelli, individua, come già detto, un paesaggio archeologico e culturale molto complesso: un territorio pluri-stratificato ${ }^{1}$ e multi- scalare (Cambi, Terrenato, 1994), fatto di una grande quantità di segni antropici e di segni naturali, elementi concreti di antiche memorie immateriali. Come sosteneva Godelier (Godelier, 1986), tutti i paesaggi archeologici che attualmente ci circondano, sono figli "dell'accumulazione dei diversi paesaggi e "passaggi" storici, che si sono succeduti con il passare del tempo, nel corso della storia". Tuttavia, la lettura e la interpretazione ontologica di questo grande "scenario", rischiano di apparire operazioni molto

complesse, se osservate con uno sguardo superficiale e frettoloso. Il paesaggio descritto nel Codice è infatti dotato di un forte carattere archeologico, per cui, per codificarlo è necessario trattarlo come se fosse un vero e proprio materiale di scavo, scomponendolo quindi nei suoi elementi primari, tangibili e misurabili. Questi elementi, che appaiono come degli iconemi (Turri, 2006), intesi come le unità elementari di percezione, sono capaci di esprimere il tutto con una funzione gerarchica primaria: attraverso gli iconemi è possibile scomporre il paesaggio in "segni", a volte "stratificati", altre volte "sovrapposti", altre volte ancora "nascosti". Il rilievo diretto e indiretto delle torri descritte dal Codice, per mezzo di metodologie miste di acquisizione 3D (laser scanner e fotogrammetria digitale) ha permesso di ottenere un dato topologico e autoptico molto preciso, non solo del singolo manufatto, ma anche (e soprattutto) di tutto quel sistema territoriale che gli sta intorno, sul quale il manufatto stesso poggia e con il quale dialoga. Le torri, dotate ognuna di una particolare giacitura e "posa archeologica" sul territorio (Fig. 6), sono state pensate e costruite per abbracciare il paesaggio da posizioni diverse, e secondo diverse modalità: da torri costruite a strapiombo sul mare, che offrono una visione panoramica della vista che si staglia dinanzi, a sistemi difensivi più isolati e strategici, oggi ormai quasi nascosti e scomparsi, poiché fagocitati da una natura brulla e selvaggia. La elaborazione di nuvole di punti, e il filtraggio dei dati, ha permesso, dunque, di decostruire il paesaggio archeologico in innumerevoli layer semantici ${ }^{2}$, con l'obiettivo di inquadrare, di volta in volta, la vista attraverso un taglio ontologico diverso. Gli strumenti digi- 
tali, infatti, oltre a fornire una lettura oggettiva e molto precisa del territorio e dei manufatti (Figg. 5 e 6), riescono ad agire su di esso come se si trattasse dello sguardo dell'archeologo (Martín Civantos, 2006): filtrando e selezionando cosa guardare, catalogandolo, interpretandolo e rendendolo disponibile per il pubblico vasto, destinatario ultimo del processo di visione, di comprensione e di compartecipazione del territorio. Tuttavia, data la natura molto variegata articolata composizione di un paesaggio archeologico, come può essere appunto quello della Calabria Ultra descritto nel Codice.

Inoltre, appare molto complesso stabilire una relazione diretta tra gli stessi elementi che costituiscono il paesaggio, poiché è difficile assegnare loro una cronologia o una gerarchia. Ciò è dato dal fatto che, nonostante l'ausilio delle moderne tecniche di indagine del territorio, $i$ dati in nostro possesso sono sempre parziali; i paesaggi archeologici e culturali che indaghiamo, infatti, altro non sono che i resti dei paesaggi storici che nel corso delle epoche si sono susseguiti. Accedere a tutto lo storico

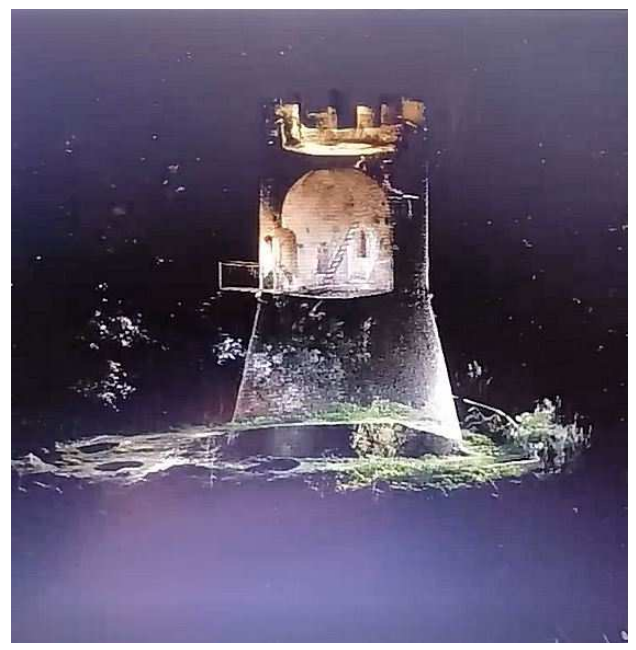

Fig. 5. Nuvola di punti dell'interno della Torre Le Pietre Nere (Palmi-Reggio Calabria).

stratigrafico sembra, quindi, quasi impossibile. Come asserisce Saramago (Saramago, 2009) è necessario rifiutare con veemenza, la possibilità di descrivere la complessità e la mutevolezza del paesaggio culturale, a causa della sua complessa mutevolezza, poiché vengono meno tutti i dati sensoriali e mnemonici di chi ha vissuto quei paesaggi in una data epoca.

Il paesaggio, in conclusione, nonostante, il nostro sguardo indagatore sia molto accurato, riesce comunque a preservare il suo fascino, a celare il proprio mistero, a eludere il nostro sguardo, e nuovamente come in uno scavo archeologico, si rivela a noi come quel grande giacimento di memoria, solo parzialmente accessibile.

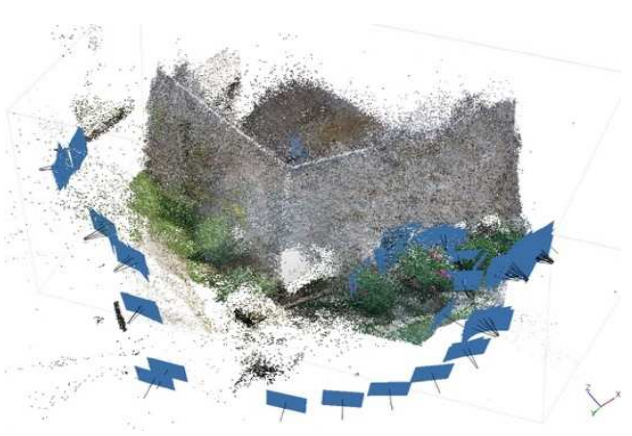

Fig. 6. Rilievo fotogrammetrico, Torre Ruffa, Ricadi (VV). Rilievo laser scanner, Fatta/Patané/Marraffa.

\section{Conclusioni: dall'analisi ontologica del pae- saggio alla narrazione del Codice: vedere, in- terpretare, comunicare}

Come detto, il paesaggio descritto dal Codice è il prodotto della sovrapposizione di processi naturali e culturali, spazialmente molto complessi e cronologicamente molto dilatati. La sua comprensione è influenzata, oltre che dall'osservazione, anche dalla percezione e dalla conoscenza degli osservatori che interpretano il territorio.

Per comprendere realmente il paesaggio archeologico descritto dal Codice, caratterizzato dalla sua complessa, ma altrettanto invisibile stratigrafia, è necessario partire dai dati digitali (Fig. 7) in nostro possesso (indagini fotogrammetriche dell'orografia dei luoghi, della struttura dei manufatti, delle sequenze degli spazi, dei confini, dell'impianto geometrico, dei linguaggio formale, etc.) e tradurre quegli stessi dati quantitativi, estratti dalle nuvole di punti, in un nuovo linguaggio di "lettura qualitativa" (Fig. 8), mnemonica e simbolica dello spazio (Criado, Boado, 1997). 


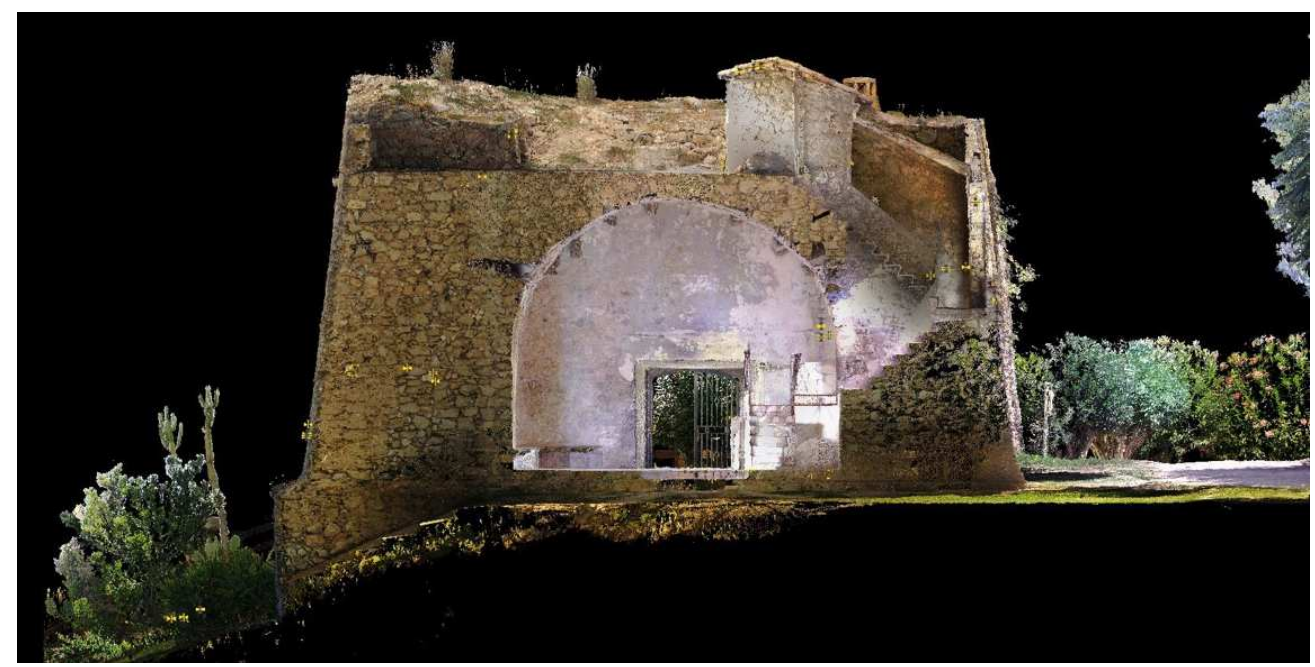

Fig. 7. Stratigrafia muraria, Torre Ruffa, Ricadi (VV). Rilievo laser scanner, Fatta/Patané/Marraffa, 2019.

Questo è possibile grazie alla elaborazione di un codice multiplo di leggibilità, comune a tutti i manufatti e comune anche allo spazio su cui essi si trovano. Tale "nuovo codice" permette di definire una struttura gerarchica dell'intero territorio costiero calabrese, classificando il paesaggio del Codice, antico e attuale, non solo secondo le sue complesse caratteristiche tipologiche, cronologiche spaziali, ma anche da un punto di vista sinestetico, proprio del racconto.

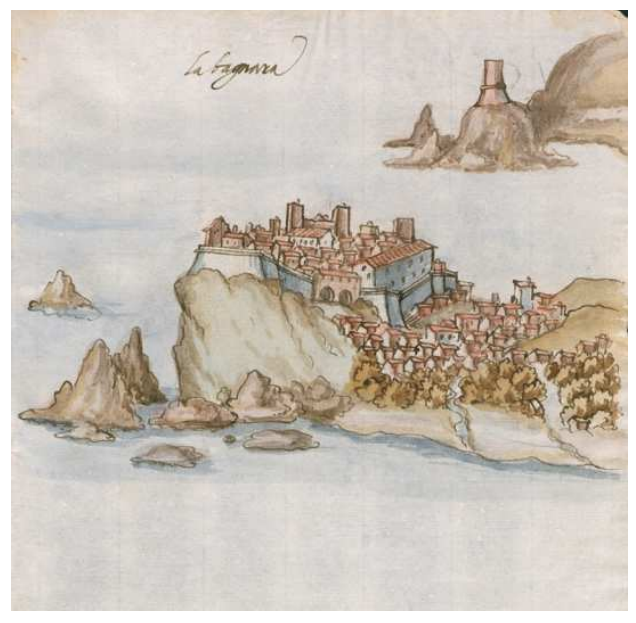

Fig. 8. C.R.C., La Bagnara, disegnata e trascritta dall'anonimo autore, XVI secolo.

La catalogazione digitale del dato, la comparazione quantitativa e qualitativa dei rilievi e la de- finizione di un vocabolario semantico (Cataldo, Rinaldi, 2007) del Codice Romano Carratelli, permetterà dunque, da una parte di ridefinire gli iconemi già esistenti e di classificarli, dall'altra di costruire dei nuovi iconemi del paesaggio archeologico, agevolando la lettura e il racconto del territorio e della sua storia secondo una prospettiva molto più ampia, incarnando il genius loci del territorio, arricchito "visivamente" di una forte carica semantica. Sembra necessario, dunque: - saper vedere, affinando il proprio sguardo $^{3}$, come se fosse quello di un archeologo; - saper interpretare, segmentando semanticamente e ontologicamente il territorio e i manufatti che agiscono su di esso; - saper comunicare, per creare modalità sempre più accattivanti e partecipative di disseminazione e conoscenza del paesaggio culturale, in cui i fruitori possano diventare allo stesso tempo attori e spettatori del messaggio culturale ${ }^{4}$, offrendo nuovi punti di fruizione sensoriale dello spazio e della storia.

Il paesaggio del Codice diventa in questo modo un grande teatro (Turri, 2006), un sistema aperto, un palinsesto di memorie in costante trasformazione interpretativa. Esso è un avvenimento, proiezione della storia, realtà polisemica, multifocale e multi-scalare, interpretato e interpretabile (Lenclud, 1995). Si propone, di conseguenza, un racconto stratigrafico digitale e analogico dei paesaggi del Codice, che mira alla sensibilità e 
al coinvolgimento emotivo, oltre che fisico, di chi legge e di chi interpreta il territorio ${ }^{5}$. La sensibilità del paesaggio archeologico, la sua potenza a "percepire e sentire attraverso i sensi", dando luogo, in una maniera più articolata, a immagini e memorie ${ }^{6}$, è caratterizzata dalla soggettività dei tempi, dei modi e soprattutto della sensibilità del fruitore. Nei paesaggi del Codice, sarà dunque possibile ri-vedere e ri-trovare il riflesso della nostra azione sul territorio, la misura del nostro vivere e modificare lo spazio che ci circonda. Il paesaggio archeologico descritto dal Codice, così inteso, appare come un libro o una lavagna, oppure ancora, come una scenografia o una grande piéce teatrale (Turner, 1986), le cui parole scritte devono essere interpretate per mezzo della conoscenza di codici e di linguaggi del tutto nuovi (Assunto, 1973).

Questo modo di ri-abbracciare il territorio, di rifarsi spettatori consapevoli e impegnati nel pro- cesso di comprensione della realtà, appare un processo lento ma inevitabile. Il perenne dualismo del paesaggio-teatro di declina in una continua interazione tra l'uomo che guarda e l'uomo che agisce, tra lo sguardo che comprende e il pensiero che seleziona, tra l'attore che interpreta e lo spettatore che contempla. (Turri, 2006).

\section{Note}

${ }^{1}$ In un paesaggio archeologico sono presenti una moltitudine di elementi, tanto artificiali quanto naturali, differenti di volta in volta nei loro attributi e caratteristiche. Per tale ragione, il paesaggio culturale si definisce un 'paesaggio pluristratigrafico, cfr. Cambi, Terrenato, 1994.

${ }^{2}$ L'annotazione semantica sulle nuvole di punti può aiutarci a leggere un tipo di paesaggio archeologico dotato di una leggibilità multi-strato e multi-livello, multi-focale, cfr. Gruber, 1993.

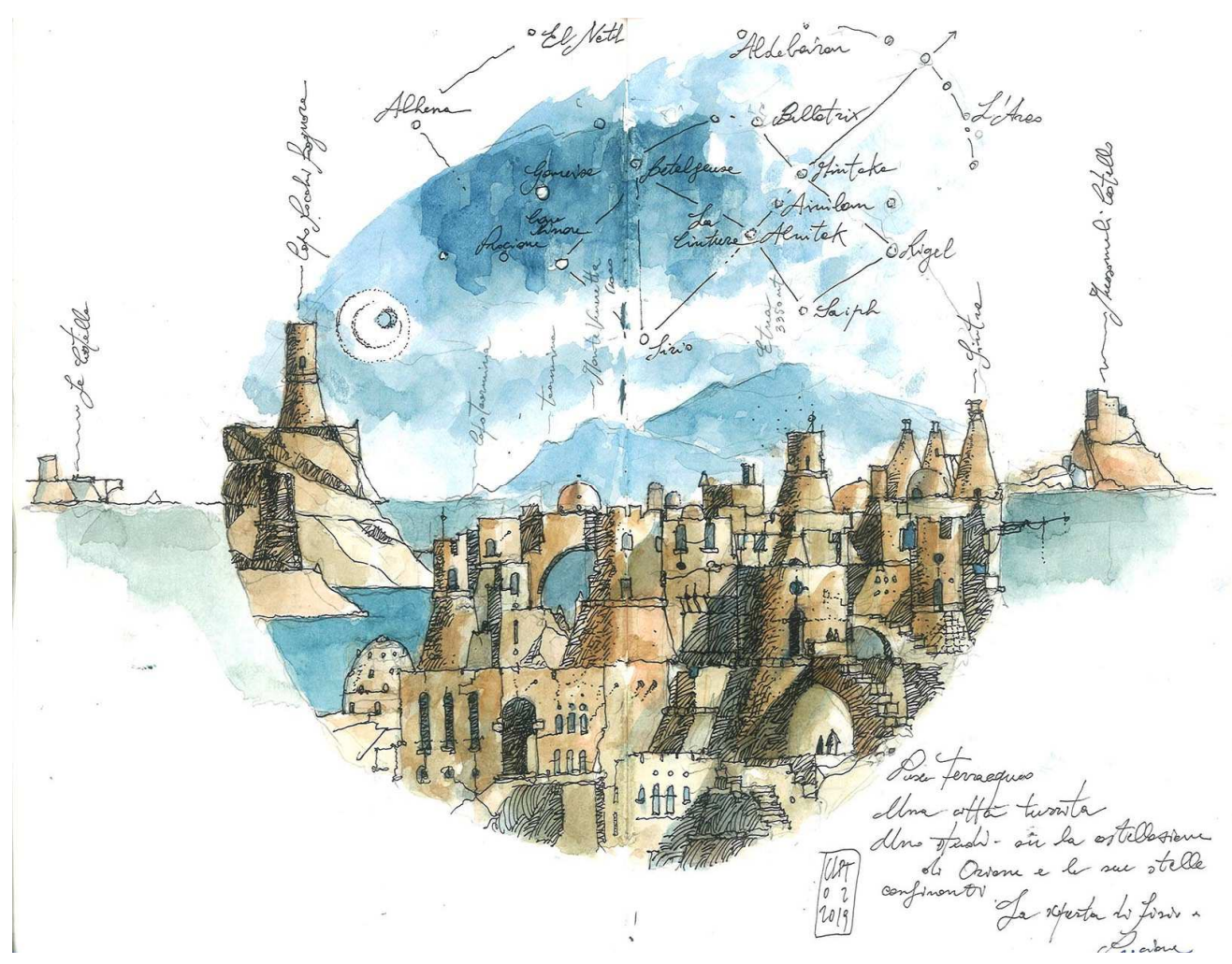

Fig. 9. "Lo Stretto e la città turrita", disegno di C. Patanè. Inchiostro e acquarello su taccuino da viaggio, 2019. 
${ }^{3}$ Gerard Lenclud, considera il paesaggio come uno spazio che viene definito dallo sguardo. Lo sguardo scompone e seleziona il paesaggio, secondo una configurazione di tipo visiva e secondo uno "statuto ontologico". Per approfondire, cfr. Lenclud, 1995.

${ }^{4}$ Espressione da intendersi come uno stimolo per lo sviluppo di una coscienza ulteriore per ogni fruitore di paesaggio culturale: un motivo per continuare un'azione all'intero di un luogo, sia da attori che da spettatori, cfr. Turri, 1998.
${ }^{5}$ L'interpretazione dei segni del paesaggio non va limitata al solo riconoscimento dei singoli elementi (attraverso una prassi di scomposizione), ma essa deve essere relazionata al contesto cui appartengono i segni stessi (rapporto col tutto), ovvero ai modi cui assumono funzionalità e significato, secondo una veste semiotica e semantica, cfr. Turri, 1979.

${ }^{6} \mathrm{Si}$ evidenza la prepotente necessità di creare ricordi, il segreto anelito di vedere il paesaggio culturale come grande contenitore di memorie territoriali, cfr. Lowenthal, 1985.

\section{Bibliography}

Abalos, I. (2005). Atlas pintoresco Vol.1: el observatorio, G.Gili, Barcelona.

Assunto, R. (1973). Il paesaggio e l'estetica, Giannini, Napoli.

Cataldo, A.; Rinaldi A.M. (2007) Using an Ontology-based Model for Knowledge Representation. In Rural Landscape, 2nd International Workshop on Ontologies for urban development: conceptual models for practitioners, Turin, Italy.

Farinelli, F. (1991). L'arguzia del paesaggio, Casabella, Milano, pp. 575-576.

Gambi, L. (1984). Introduzione, in Archivio di Stato, L'immagine integrata, Milano.

Gruber, T.R. (1993). "A translation approach to portable ontology specifications”, Knowl Acquis, 5, pp. 199-220.

Lenclud, G. (1995). "Ethnologie et paysage", in Voisenat, C. ed., Paysage au pluriel. Pour une aproche ethnologique des paysages, Édition de la Maison des Sciences de l'Homme, Paris.

Marini, S. (2006). “Cit. Renato Bocchi”, in Dessiner sur l'herbe, Il Poligrafo Ed., Padova.

Martín Civantos, J. (2006). "Published in Francovich", in Marco, R.; Marco, V., eds., Atti del IV Congresso nazionale di Archeologia Medievale, Firenze, pp. 3-8.

Romano, G. (1978). Studi sul paesaggio, Einaudi, Torino.

Turner, V. (1986). Dal rito al teatro, il Mulino, Bologna.

Turri, E. (1979). Semiologia del paesaggio italiano, Longanesi, Milano.

Turri, E. (2006). Il paesaggio come teatro. Dal territorio vissuto al territorio rappresentato, Marsilio, Venezia.

VV.AA. (1991). Il disegno del paesaggio italiano, Gregotti, V. ed., Casabella, Milano. 